\title{
Pension funding incorporating downside risks
}

\author{
S.C. Chang ${ }^{\text {a }}$, Larry Y. Tzeng ${ }^{b, *}$, Jerry C.Y. Miao ${ }^{c}$ \\ a Department of Risk Management and Insurance, National Chengchi University, Taipei, Taiwan, ROC \\ ${ }^{\mathrm{b}}$ Department of Finance, National Taiwan University, 50 Lane 144, Sec. 4 Keelung Road, Taipei, Taiwan, ROC \\ ${ }^{\mathrm{c}}$ Department of Insurance, Tamkang University, Taipei, Taiwan, ROC
}

Received 1 April 2002; received in revised form 1 September 2002; accepted 12 December 2002

\begin{abstract}
This research extends Haberman and Sung's [Insurance: Mathematics and Economics 15 (1994) 151] and Chang's [Insurance: Mathematics and Economics 24 (1999) 187] works to study optimal funding strategies through the control mechanism. The paper further generalizes the previous research in three ways. First, downside risks, under-funding risk and over-contributing risk, are included additionally in the risk minimization criterion to obtain the optimal solutions. Second, we allow the weighting factors in the performance criterion to belong to a broader parametric family. Third, the rates of investment returns are assumed to follow the auto-regressive process. The above three generalization indeed include traditional model as special cases. Furthermore, an actual case is employed to investigate their financial impacts on funding and contribution due to our generalization. The results show that neglecting to recognize the under-funding risk and the over-contribution risk will lead to a significant difference in optimal funding schedule. The weighting factors and the returns of investment also play critical roles in obtaining the optimal strategy.
\end{abstract}

() 2003 Elsevier Science B.V. All rights reserved.

Keywords: Optimal pension funding; Under-funding; Over-contributing

\section{Introduction}

A defined-benefit pension fund confronts uncertainties from both demographic and economic shifts, such as wage structure, turnover rate, inflation rate and interest rate. It is well known that these demographic and financial variables may be guided by stochastic processes rather than given as a deterministic assumption. Hence, a control mechanism has been proposed to link together stochastic simulations and equation-type optimal solutions, thus providing an efficient way for evaluating the risk effects caused by the specific plan strategies. The stochastic modeling of the fund dynamics could provide helpful guidance for proper assessment of the trade-off between various risks along the investment time horizon. Hence, the future fund dynamics could be measured properly by this approach. Studies on pension funding in recent decades can be found in Bowers et al. (1982), McKenna (1982), Dufresne (1988, 1989), Haberman (1992-1994), Mandl and Mazurova (1996), Gerrard and Haberman (1996), Haberman and Wong (1997), Cairns and Parker (1997) and Owadally and Haberman (1999, 2000). To capture the stochastic nature of pension funds, many researchers (Benjamin, 1984, 1989; O’Brian, 1986, 1987; Vanderbroek, 1990; Haberman, 1993, 1994;

\footnotetext{
* Corresponding author.

E-mail address: tzeng@ms.cc.ntu.edu.tw (L.Y. Tzeng).
} 
Haberman and Sung, 1994; Chang, 1999) proposed using the control theory when analyzing the funding strategy of a pension under stochastic environments.

Daykin et al. (1994, Chapter 16) outlined a practical simulation procedure in modeling the pension dynamics. They discussed the valuation assumptions on age and time scaling, aging and renewing cohort, pay in pension, benefits, and contributions. They also discussed both deterministic and stochastic methods of pension funding. When further characterizing the objective of a pension fund, Haberman and Sung (1994) and Chang (1999) recognized two major risks, contribution rate risk and solvency risk. Our paper is an extension of their work and intends to provide additional contributions in three ways.

First, because previous researchers used square of deviation in their performance criterion function, they weighted implicitly under-funding as over-funding and over-contribution as under-contribution. In Haberman and Sung (1994), the contribution risk is measured by the square of deviation between the employer's contribution and target contribution rate, while the solvency risk is measured by the square of deviation between pension fund's assets and target liabilities. Instead of using the square of amount deviation, Chang (1999) proposed measuring the contribution risk and solvency risk by the square of ratio deviation. However, a pension fund manager may care more about under-funding than over-funding, and over-contribution than under-contribution. This study intends to overcome this problem by revising the performance criterion function of pension funds. In addition to the contribution risk and solvency risk, we add two additional components of risks, under-funding risk and over-contribution risk.

Second, we rearrange the performance criterion function by allowing various weighting factors that can be adjusted by the decision-maker's preference on time and risk. To reflect the time preference of the decision maker, both Haberman and Sung (1994) and Chang (1999) aggregate the components at different time periods by $1 /(1+\text { interest rate })^{\text {time period }}$, which is commonly used to aggregate cash flows at different periods. However, the components in their performance criterion function are indeed square of deviation rather than cash flow. A legitimate question can be raised is whether we still employ $1 /(1+\text { interest rate })^{\text {time period }}$ as discount factors when the components in the performance criterion function are indeed square of deviation rather than cash flow. In fact, the performance criterion plays a role like dis-utility of the decision maker. Generally, as in most economy research, the discount factor can be expressed as $1 /(1+\text { discount rate })^{\text {time period }}$ to reflect decision-maker's time preference, and, moreover, discount rate may not be always equal to interest rate. On the other hand, the weighting factors may not only represent decision-maker's preference on time but could also reflect decision-maker's preference on risk. For example, if the government prohibits under-funding, then we may need to assign a relatively high value on solvency risk. This paper proposes that the decision maker should determine weighting factors and it is not necessary to use interest rates as discount rates. Moreover, we demonstrate that the general model can be reduced to that of Haberman and Sung (1994) and Chang (1999) simply by assigning specific weighting factors.

Third, rate of investment return at each period following independent identical distribution is assumed in previous works. However, many papers show that investment return may exhibit auto-correlation pattern over time. Our paper adopts Haberman's approach by assuming auto-regressive rates of investment return. Because the performance criterion function, the weighting factors, and return process used in our paper can be regarded as a generalization of the model proposed in Haberman and Sung (1994) and Chang (1999), our model include theirs as special cases.

Furthermore, we use an actual case to investigate the impact of these risk components on the contribution of a pension fund. Stochastic procedures using time as the operational parameter are employed to obtain the best estimates of the projected workforce, while the cash flows characterizing the plan liability are scrutinized through dynamic simulations. ${ }^{1}$ We find that neglecting to recognize under-funding risk and over-contribution risk in pension fund may have a significant impact on funding strategy. The assumptions of the weighting factors and rates of investment return play critical roles in management of pension funding.

In the next section, we outline the proposed model and the optimal solutions. In Section 3, our model is applied to an actual case and the results are discussed. The conclusions follow in the last section.

\footnotetext{
${ }^{1}$ For details on these, see Bacinello (1988) and Chang (1999).
} 


\section{Model}

\subsection{Optimal contribution}

As in Chang (2000), we assume that $C_{s}, \mathrm{NC}_{s}, F_{s}$, and $\mathrm{AL}_{s}$ are contribution, normal cost, fund asset, and accrued liability of a pension fund at time $s$, respectively. Haberman and Sung (1994) and Chang (1999) recognized two main risks in a pension fund, contribution risk and solvency risk. Let $\alpha_{1, s}\left(C_{s} / \mathrm{NC}_{s}-1\right)^{2}$ and $\alpha_{2, s+1}\left(1-F_{s+1} / \eta \mathrm{AL}_{s+1}\right)^{2}$ denote the contribution risk and solvency risk, where $\eta$ is target fund ratio, and $\alpha_{1, s}$ and $\alpha_{2, s}$ are weighting factors for the contribution risk and solvency risk at time $s$, respectively.

However, these quadratic terms, $\alpha_{1, s}\left(C_{s} / \mathrm{NC}_{s}-1\right)^{2}$ and $\alpha_{2, s+1}\left(1-F_{s+1} / \eta \mathrm{AL}_{s+1}\right)^{2}$, do not differentiate between under-funding and over-funding, nor do between over-contribution and under-contribution. Indeed, under-funding and/or over-contribution may be the main concern of a pension fund manager. To measure these types of asymmetry risks, we propose using $\alpha_{3, s}\left(C_{s} / \mathrm{NC}_{s}-1\right)$ and $\alpha_{4, s+1}\left(1-F_{s+1} / \eta \mathrm{AL}_{s+1}\right)$ to evaluate the over-contribution risk and under-funding risk, where $\alpha_{3, s}$ and $\alpha_{4, s}$ are weighting factors for the over-contribution rate risk and under-funding risk at time $s$, respectively. Thus, performance criteria $J$ of a pension fund can be expressed as

$$
J=\sum_{s=t}^{T-1} \alpha_{1, s}\left(\frac{C_{s}}{\mathrm{NC}_{s}}-1\right)^{2}+\alpha_{2, s+1}\left(1-\frac{F_{s+1}}{\eta \mathrm{AL}_{s+1}}\right)^{2}+\alpha_{3, s}\left(\frac{C_{s}}{\mathrm{NC}_{s}}-1\right)+\alpha_{4, s+1}\left(1-\frac{F_{s+1}}{\eta \mathrm{AL}_{s+1}}\right)
$$

To demonstrate that Eq. (1) includes the models of both Haberman and Sung (1994) and Chang (1999) as special case, let $v^{s}$ (or $v_{s}$ ) and $\beta_{s}$ denote discount factor and risk weighted ratio at time $s$, respectively, as Haberman and Sung (1994) and Chang (1999). The discount factor and risk weighted ratio are components of weighting factors in our paper. The performance criterion function in Haberman and Sung (1994) is Eq. (1) with $\alpha_{1, s}=v^{s} \mathrm{NC}_{s}^{2}$, $\alpha_{2, s+1}=v^{s+1} \beta_{s+1} \eta^{2} \mathrm{AL}_{t+1}^{2}, \alpha_{3, s}=0$, and $\alpha_{4, s}=0$, while that in Chang (1999) is the case with $\alpha_{1, s}=v_{s}$, $\alpha_{2, s+1}=v_{s+1} \beta_{s+1}, \alpha_{3, s}=0$, and $\alpha_{4, s}=0$.

On the other hand, assume that $F_{s+1}=\left(F_{s}+C_{s}-B_{s}\right)\left(1+r_{s+1}\right)$, where $B_{s}$ is benefit outgo for time $s$ and $r_{s+1}$ is the gross rate of investment return of the pension fund at time $s+1$. Haberman and Sung (1994) and Chang (1999) regarded the investment returns as independent through time, but AR(1) models have been considered by Haberman (1994), Mandl and Mazurova (1996), Cairns and Parker (1997). For generality, we assume that the return rate of pension fund follows a auto-regressive process: $r_{s+1}=\theta+\kappa\left(r_{s}-\theta\right)+\varepsilon_{s}$, where $\theta$ and $\kappa$ are constants and $\varepsilon_{s}$ follows $\operatorname{Normal}\left(0, \sigma^{2} r_{s}\right)$. The assumption of independent identical distribution is the special case when $\kappa=0$. Of course, whether the auto-regressive process is a better assumption than the independent process depends on the situation pension managers cope with and should be evaluated by empirical evidences. Our paper focuses on showing the stochastic method of pension funding can be extended to the case under auto-regressive process.

Following the proposed algorithm (see also Haberman and Sung, 1994, p. 158; Chang, 1999, p. 193), we can formulate the optimization of our model as

$$
\min _{C_{t}, \ldots, C_{T-1}} E\left[J \mid F_{t}, r_{t}\right] \quad \text { s.t. } \quad\left\{\begin{array}{l}
F_{t+1}=\left(F_{t}+C_{t}-B_{t}\right)\left(1+r_{t+1}\right) \\
r_{t+1}=\theta+\kappa\left(r_{t}-\theta\right)+\varepsilon_{t} \\
\varepsilon_{t} \sim N\left(0, \sigma^{2} r_{t}\right)
\end{array}\right.
$$

To proceed by induction, we define $V_{t}\left(F_{t}, r_{t}\right)$ as

$$
V_{t}\left(F_{t}, r_{t}\right)=\min _{C_{t}, \ldots, C_{T-1}} E\left[J \mid F_{t}, r_{t}\right]
$$


For the principle of optimality, we have the Bellman equation: ${ }^{2}$

$$
\begin{gathered}
V_{t}\left(F_{t}, r_{t}\right)=\min _{C_{t}} E\left\{\alpha_{1, t}\left(\frac{C_{t}}{\mathrm{NC}_{t}}-1\right)^{2}+\alpha_{2, t+1}\left(1-\frac{F_{t+1}}{\eta \mathrm{AL}_{t+1}}\right)^{2}+\alpha_{3, t}\left(\frac{C_{t}}{\mathrm{NC}_{t}}-1\right)\right. \\
\left.+\alpha_{4, t+1}\left(1-\frac{F_{t+1}}{\eta \mathrm{AL}_{t+1}}\right)+V_{t+1}\left(F_{t+1}, r_{t+1}\right) \mid F_{t}, r_{t}\right\} .
\end{gathered}
$$

We set $V_{T}\left(F_{T}, r_{T}\right)=0$ as a boundary condition for there is no expected loss associated with the terminal state. Since we add in a linear component in the performance criterion, we still try the solution of Eq. (4) by quadratic form $^{3}$ which includes linear function as a special case. Let $V_{t}\left(F_{t}, r_{t}\right)=a_{1, t}\left(r_{t}\right) F_{t}^{2}+a_{2, t}\left(r_{t}\right) F_{t}+a_{3, t}\left(r_{t}\right)$ for all $t, t \in[0, T]$. It should be noticed that $a_{1, t}\left(r_{t}\right), a_{2, t}\left(r_{t}\right)$ and $a_{3, t}\left(r_{t}\right)$ are coefficients. ${ }^{4}$ Hence, the Bellman equation can be rewritten as

$$
V_{t}\left(F_{t}, r_{t}\right)=\min _{C_{t}}\left\{\left(\frac{\alpha_{1, t}}{\mathrm{NC}_{t}^{2}}+\frac{\alpha_{2, t+1} K_{t+1}}{\eta^{2} \mathrm{AL}_{t+1}^{2}}+a_{1, t+1}\left(r_{t+1}\right) K_{t+1}\right) C_{t}^{2}+\mathrm{o}(\cdot)\right\},
$$

$\mathrm{o}(\cdot)$ is the other terms which are not correlated with $C_{t}^{2}$. In order to have a unique solution to $C_{t}$, the sufficient condition for $V_{t}\left(F_{t}, r_{t}\right)$ is strictly convex function to $C_{t}$ is

$$
a_{1, t+1}\left(r_{t+1}\right)>-\left(\frac{\alpha_{1, t}}{K_{t+1} \mathrm{NC}_{t}^{2}}+\frac{\alpha_{2, t+1}}{\eta^{2} \mathrm{AL}_{t+1}^{2}}\right),
$$

where $H_{t+1}=(1+\theta)+\kappa\left(\theta-r_{t}\right), K_{t+1}=\sigma^{2} r_{t}+H_{t+1}^{2}$ are in the AR(1) process.

Then contribution can be estimated by induction. The optimal contribution $C_{t}^{*}$ is

$$
C_{t}^{*}=\frac{D_{t}+E_{t} F_{t}}{G_{t}},
$$

where

$$
\begin{aligned}
D_{t}= & \frac{2 \alpha_{1, t}}{\mathrm{NC}_{t}}+\frac{2 \alpha_{2, t+1} H_{t+1}}{\eta \mathrm{AL}_{t+1}}+\frac{2 \alpha_{2, t+1} B_{t} K_{t+1}}{\eta^{2} \mathrm{AL}_{t+1}^{2}}-\frac{\alpha_{3, t}}{\mathrm{NC}_{t}} \\
& +\frac{\alpha_{4, t+1} H_{t+1}}{\eta \mathrm{AL}_{t+1}}+2 a_{1, t+1}\left(r_{t+1}\right) B_{t} K_{t+1}-a_{2, t+1}\left(r_{t+1}\right) H_{t+1}, \\
E_{t}= & \frac{-2 \alpha_{2, t+1} K_{t+1}}{\eta^{2} \mathrm{AL}_{t+1}^{2}}-a_{1, t+1}\left(r_{t+1}\right) K_{t+1}, \\
G_{t}= & \frac{2 \alpha_{1, t}}{\mathrm{NC}_{t}^{2}}+\frac{2 \alpha_{2, t+1} K_{t+1}}{\eta^{2} \mathrm{AL}_{t+1}^{2}}+2 a_{1, t+1}\left(r_{t+1}\right) K_{t+1} .
\end{aligned}
$$

The recursive relationships for $a_{1, t}\left(r_{t}\right)$ and $a_{2, t}\left(r_{t}\right)$ are solved by the following equations, respectively:

$$
a_{1, t}\left(r_{t}\right)=\frac{\alpha_{1, t} E_{t}^{2}}{G_{t}^{2} \mathrm{NC}_{t}^{2}}+\frac{\alpha_{2, t+1}\left(G_{t}+E_{t}\right)^{2} K_{t+1}}{G_{t}^{2} \eta^{2} \mathrm{AL}_{t+1}^{2}}+\frac{a_{1, t+1}\left(r_{t+1}\right)\left(G_{t}+E_{t}\right)^{2} K_{t+1}}{G_{t}^{2}},
$$

\footnotetext{
${ }^{2}$ Our main interest is in discussing the optimal contributions, so to avoid further technicalities we assume there exists a unique control satisfying the Bellman equation. Please refer the explanations in Cox et al. (1985, p. 370).

${ }^{3}$ Since we add in two linear components as additional risks, these terms only make the optimal solution paths shift but preserve the form of the optimal solution.

${ }^{4}$ The notations of $a_{1, t}\left(r_{t}\right), a_{2, t}\left(r_{t}\right)$ and $a_{3, t}\left(r_{t}\right)$ denote coefficients rather than the product of two values.
} 


$$
\begin{aligned}
a_{2, t}\left(r_{t}\right)= & \frac{-2 \alpha_{1, t} E_{t}}{G_{t} \mathrm{NC}_{t}}\left(1-\frac{D_{t}}{G_{t} \mathrm{NC}_{t}}\right)-\frac{2 \alpha_{2, t+1} H_{t+1}\left(G_{t}+E_{t}\right)}{\eta \mathrm{AL}_{t+1} G_{t}}+\frac{2 \alpha_{2, t+1} K_{t+1}\left(G_{t}+E_{t}\right)\left(D_{t}-B_{t} G_{t}\right)}{\eta^{2} \mathrm{AL}_{t+1}^{2} G_{t}^{2}} \\
& +\frac{\alpha_{3, t} E_{t}}{G_{t} \mathrm{NC}_{t}}-\frac{\alpha_{4, t+1} H_{t+1}\left(G_{t}+E_{t}\right)}{\eta \mathrm{AL}_{t+1} G_{t}}+\frac{2 a_{1, t+1}\left(r_{t+1}\right) K_{t+1}\left(G_{t}+E_{t}\right)\left(D_{t}-B_{t} G_{t}\right)}{G_{t}^{2}} \\
& +\frac{a_{2, t+1}\left(r_{t+1}\right) H_{t+1}\left(G_{t}+E_{t}\right)}{G_{t}} .
\end{aligned}
$$

Since the boundary condition is $V_{T}\left(F_{T}, r_{T}\right)=0$,

$$
a_{1, T}\left(r_{T}\right)=0, \quad \text { and } \quad a_{2, T}\left(r_{T}\right)=0 .
$$

The optimal solution is similar to those derived by Haberman and Sung (1994) and Chang (1999), except for the additional terms for downside risks. On the other hand, since Haberman and Sung (1994) and Chang (1999) assume that the rate of investment return follows independent identical distribution, then $H_{t+1}=1+\theta$ and $K_{t+1}=\sigma^{2}+H_{t+1}^{2}$ in their models, which is a special case when $\kappa=0$.

\subsection{Decomposition of contribution}

From Eqs. (8)-(12), we can find that $\alpha_{3, s}$ and $\alpha_{4, s}$ exist only in the equations for $a_{2, t}\left(r_{t}\right)$ and $D_{t}$, and have no influence on equations for $a_{1, t}\left(r_{t}\right), E_{t}$ or $G_{t}$. Thus, we can conclude that the additional terms for downside risks affect the optimal contribution through Eqs. (8) and (12). Substituting (8) into (7), we can get the following equation:

$$
\begin{aligned}
C_{t}^{*}= & \left\{\frac{2 \alpha_{1, t}}{G_{t} \mathrm{NC}_{t}}+\frac{2 \alpha_{2, t+1} H_{t+1}}{G_{t} \eta \mathrm{AL}_{t+1}}+\frac{2 \alpha_{2, t+1} B_{t} K_{t+1}}{G_{t} \eta^{2} \mathrm{AL}_{t+1}^{2}}+\frac{2 a_{1, t+1}\left(r_{t+1}\right) B_{t} K_{t+1}}{G_{t}}+\frac{E_{t}}{G_{t}} F_{t}\right\} \\
& +\left\{-\frac{a_{2, t+1}\left(r_{t+1}\right) H_{t+1}}{G_{t}}\right\}+\left\{-\frac{\alpha_{3, t}}{G_{t} \mathrm{NC}_{t}}+\frac{\alpha_{4, t+1} H_{t+1}}{G_{t} \eta \mathrm{AL}_{t+1}}\right\} \\
= & \{\text { fixed component }\}+\{\text { long-term effect }\}+\{\text { short-term effect }\} .
\end{aligned}
$$

$C_{t}$ can be decomposed into three components with respect to the impact of $\alpha_{3, t}$ and $\alpha_{4, t}$. The first part of $C_{t}$ is the fixed component that is not affected by $\alpha_{3, t}$ or $\alpha_{4, t}$. The second part of $C_{t}$ is the long-term effect of $\alpha_{3, t}$ or $\alpha_{4, t}$ affected through $a_{2, t}\left(r_{t}\right)$ which is a recursive term from $T$ to $t$. If we have $\alpha_{3, s}>0$ or $\alpha_{4, s}>0$ for any $s>t$, $a_{2, t}\left(r_{t}\right)$ will have $\alpha_{3, s}$ or $\alpha_{4, s}$ term. It means the contribution at time $t$ should do some adjustment for minimizing future risks. The third part of $C_{t}$ is the short-term effect affected by $\alpha_{3, t}$ and $\alpha_{4, t}$. It is obvious that the short-term effect of downside risks always increases (decreases) optimal contributions when $\alpha_{4, t}\left(\alpha_{3, t}\right)$ is positive. However, the result of the net effect has to be determined by further simulation.

\section{Results and analysis}

We use the data in Chang $(1999)^{5}$ to illustrate these results. The estimated actuarial accrued liabilities, normal costs and benefit payments are generated under the following assumptions:

- Population: Tai-PERS service table based on 1995-1996; 1989 TSO for the retiree's annuity table.

- Number of employees in the sample: 3823 .

- Actuarial cost method: individual entry age normal cost method.

\footnotetext{
5 The data is publicly available and can be directly retrieved from Chang (1999). For more demographic characteristics of the sample, please refer Chang $(1999,2000)$ and Chang and Chen (2002). Please also find the data description of Tai-PERS, the basic actuarial assumptions and the demographic information in Section 3 [Taiwan's Public Employees Retirement System] and Section 4 [Application of the Methodology to Tai-PERS] in Chang (2000) and also the discussions in the "Numerical Experiments" in Chang and Cheng (2002).
} 
Table 1

Contribution ratios and $\beta$ (for initial fund ratios of $0.8,1.0,1.2)^{\mathrm{a}}$

\begin{tabular}{|c|c|c|c|c|c|c|c|c|c|}
\hline \multirow[t]{2}{*}{ Time } & \multicolumn{3}{|c|}{$\beta_{3}=0 ; \beta_{4}=0$} & \multicolumn{3}{|c|}{$\beta_{3}=0 ; \beta_{4}=0.5$} & \multicolumn{3}{|c|}{$\beta_{3}=0 ; \beta_{4}=1.0$} \\
\hline & 0.8 & 1.0 & 1.2 & 0.8 & 1.0 & 1.2 & 0.8 & 1.0 & 1.2 \\
\hline 1 & 1.330 & 1.261 & 1.198 & 1.577 & 1.509 & 1.446 & 1.826 & 1.760 & 1.693 \\
\hline 2 & 1.302 & 1.261 & 1.224 & 1.509 & 1.468 & 1.424 & 1.715 & 1.670 & 1.634 \\
\hline 3 & 1.303 & 1.275 & 1.246 & 1.482 & 1.454 & 1.428 & 1.661 & 1.636 & 1.601 \\
\hline 4 & 1.292 & 1.270 & 1.248 & 1.449 & 1.429 & 1.405 & 1.607 & 1.584 & 1.564 \\
\hline 5 & 1.276 & 1.259 & 1.244 & 1.419 & 1.399 & 1.383 & 1.557 & 1.542 & 1.524 \\
\hline 6 & 1.266 & 1.250 & 1.237 & 1.386 & 1.375 & 1.361 & 1.512 & 1.499 & 1.485 \\
\hline 7 & 1.250 & 1.239 & 1.228 & 1.362 & 1.349 & 1.338 & 1.471 & 1.459 & 1.449 \\
\hline 8 & 1.236 & 1.226 & 1.219 & 1.334 & 1.324 & 1.317 & 1.433 & 1.423 & 1.412 \\
\hline 9 & 1.220 & 1.212 & 1.204 & 1.306 & 1.298 & 1.289 & 1.391 & 1.383 & 1.377 \\
\hline 10 & 1.199 & 1.192 & 1.186 & 1.275 & 1.267 & 1.261 & 1.352 & 1.345 & 1.337 \\
\hline 11 & 1.176 & 1.169 & 1.165 & 1.241 & 1.236 & 1.229 & 1.306 & 1.300 & 1.295 \\
\hline 12 & 1.155 & 1.150 & 1.145 & 1.211 & 1.205 & 1.201 & 1.267 & 1.261 & 1.257 \\
\hline 13 & 1.132 & 1.128 & 1.125 & 1.180 & 1.176 & 1.172 & 1.228 & 1.223 & 1.219 \\
\hline 14 & 1.113 & 1.109 & 1.106 & 1.152 & 1.149 & 1.145 & 1.192 & 1.188 & 1.184 \\
\hline 15 & 1.092 & 1.089 & 1.088 & 1.125 & 1.122 & 1.119 & 1.157 & 1.154 & 1.151 \\
\hline 16 & 1.074 & 1.071 & 1.069 & 1.099 & 1.097 & 1.095 & 1.125 & 1.122 & 1.120 \\
\hline 17 & 1.056 & 1.054 & 1.052 & 1.076 & 1.074 & 1.071 & 1.095 & 1.093 & 1.090 \\
\hline 18 & 1.038 & 1.036 & 1.035 & 1.051 & 1.050 & 1.049 & 1.065 & 1.063 & 1.062 \\
\hline 19 & 1.021 & 1.019 & 1.019 & 1.029 & 1.028 & 1.026 & 1.037 & 1.036 & 1.034 \\
\hline \multirow[t]{3}{*}{20} & 1.004 & 1.004 & 1.003 & 1.008 & 1.007 & 1.006 & 1.011 & 1.010 & 1.009 \\
\hline & \multicolumn{3}{|c|}{$\beta_{3}=0.5 ; \beta_{4}=0$} & \multicolumn{3}{|c|}{$\beta_{3}=0.5 ; \beta_{4}=0.5$} & \multicolumn{3}{|c|}{$\beta_{3}=0.5 ; \beta_{4}=1.0$} \\
\hline & 0.8 & 1.0 & 1.2 & 0.8 & 1.0 & 1.2 & 0.8 & 1.0 & 1.2 \\
\hline 1 & 1.242 & 1.179 & 1.109 & 1.494 & 1.427 & 1.358 & 1.740 & 1.674 & 1.611 \\
\hline 2 & 1.195 & 1.154 & 1.115 & 1.399 & 1.359 & 1.320 & 1.607 & 1.567 & 1.525 \\
\hline 3 & 1.181 & 1.152 & 1.122 & 1.362 & 1.332 & 1.304 & 1.538 & 1.509 & 1.481 \\
\hline 4 & 1.154 & 1.134 & 1.112 & 1.311 & 1.289 & 1.268 & 1.467 & 1.448 & 1.426 \\
\hline 5 & 1.124 & 1.108 & 1.090 & 1.266 & 1.248 & 1.231 & 1.407 & 1.389 & 1.374 \\
\hline 6 & 1.100 & 1.087 & 1.073 & 1.224 & 1.210 & 1.198 & 1.349 & 1.334 & 1.322 \\
\hline 7 & 1.076 & 1.065 & 1.053 & 1.187 & 1.175 & 1.164 & 1.296 & 1.288 & 1.276 \\
\hline 8 & 1.052 & 1.043 & 1.033 & 1.151 & 1.141 & 1.132 & 1.247 & 1.238 & 1.229 \\
\hline 9 & 1.027 & 1.019 & 1.011 & 1.113 & 1.104 & 1.098 & 1.201 & 1.193 & 1.183 \\
\hline 10 & 1.000 & 0.993 & 0.986 & 1.075 & 1.067 & 1.061 & 1.150 & 1.144 & 1.138 \\
\hline 11 & 0.969 & 0.964 & 0.958 & 1.035 & 1.029 & 1.023 & 1.100 & 1.095 & 1.088 \\
\hline 12 & 0.941 & 0.936 & 0.931 & 0.998 & 0.993 & 0.989 & 1.053 & 1.049 & 1.044 \\
\hline 13 & 0.914 & 0.911 & 0.906 & 0.962 & 0.957 & 0.952 & 1.010 & 1.006 & 1.001 \\
\hline 14 & 0.889 & 0.886 & 0.882 & 0.929 & 0.925 & 0.922 & 0.968 & 0.965 & 0.961 \\
\hline 15 & 0.864 & 0.862 & 0.859 & 0.897 & 0.894 & 0.891 & 0.929 & 0.927 & 0.924 \\
\hline 16 & 0.842 & 0.840 & 0.837 & 0.868 & 0.865 & 0.863 & 0.894 & 0.892 & 0.888 \\
\hline 17 & 0.820 & 0.819 & 0.817 & 0.840 & 0.838 & 0.836 & 0.859 & 0.857 & 0.856 \\
\hline 18 & 0.799 & 0.798 & 0.796 & 0.813 & 0.811 & 0.810 & 0.827 & 0.825 & 0.823 \\
\hline 19 & 0.779 & 0.778 & 0.777 & 0.787 & 0.786 & 0.785 & 0.795 & 0.794 & 0.793 \\
\hline 20 & 0.760 & 0.759 & 0.758 & 0.763 & 0.762 & 0.761 & 0.766 & 0.765 & 0.764 \\
\hline
\end{tabular}


Table 1 (Continued)

\begin{tabular}{|c|c|c|c|c|c|c|c|c|c|}
\hline \multirow[t]{2}{*}{ Time } & \multicolumn{3}{|c|}{$\beta_{3}=1.0 ; \beta_{4}=0$} & \multicolumn{3}{|c|}{$\beta_{3}=1.0 ; \beta_{4}=0.5$} & \multicolumn{3}{|c|}{$\beta_{3}=1.0 ; \beta_{4}=1.0$} \\
\hline & 0.8 & 1.0 & 1.2 & 0.8 & 1.0 & 1.2 & 0.8 & 1.0 & 1.2 \\
\hline 1 & 1.155 & 1.091 & 1.024 & 1.407 & 1.342 & 1.273 & 1.656 & 1.588 & 1.521 \\
\hline 2 & 1.089 & 1.047 & 1.008 & 1.293 & 1.250 & 1.212 & 1.497 & 1.458 & 1.422 \\
\hline 3 & 1.058 & 1.029 & 1.000 & 1.236 & 1.209 & 1.179 & 1.416 & 1.387 & 1.355 \\
\hline 4 & 1.018 & 0.995 & 0.971 & 1.175 & 1.153 & 1.132 & 1.334 & 1.311 & 1.289 \\
\hline 5 & 0.971 & 0.957 & 0.940 & 1.111 & 1.097 & 1.078 & 1.253 & 1.235 & 1.220 \\
\hline 6 & 0.938 & 0.922 & 0.909 & 1.062 & 1.046 & 1.033 & 1.186 & 1.171 & 1.157 \\
\hline 7 & 0.901 & 0.890 & 0.879 & 1.012 & 1.001 & 0.991 & 1.124 & 1.111 & 1.101 \\
\hline 8 & 0.869 & 0.860 & 0.849 & 0.966 & 0.957 & 0.946 & 1.065 & 1.055 & 1.042 \\
\hline 9 & 0.834 & 0.825 & 0.819 & 0.920 & 0.913 & 0.905 & 1.006 & 0.998 & 0.991 \\
\hline 10 & 0.798 & 0.792 & 0.786 & 0.875 & 0.868 & 0.861 & 0.951 & 0.944 & 0.935 \\
\hline 11 & 0.763 & 0.755 & 0.751 & 0.827 & 0.821 & 0.815 & 0.894 & 0.890 & 0.882 \\
\hline 12 & 0.729 & 0.723 & 0.718 & 0.785 & 0.780 & 0.775 & 0.840 & 0.835 & 0.829 \\
\hline 13 & 0.695 & 0.691 & 0.687 & 0.742 & 0.739 & 0.734 & 0.790 & 0.788 & 0.782 \\
\hline 14 & 0.666 & 0.662 & 0.658 & 0.705 & 0.702 & 0.698 & 0.744 & 0.741 & 0.736 \\
\hline 15 & 0.637 & 0.634 & 0.631 & 0.669 & 0.666 & 0.663 & 0.701 & 0.700 & 0.695 \\
\hline 16 & 0.610 & 0.607 & 0.605 & 0.636 & 0.634 & 0.631 & 0.662 & 0.659 & 0.656 \\
\hline 17 & 0.585 & 0.583 & 0.581 & 0.604 & 0.602 & 0.600 & 0.623 & 0.622 & 0.620 \\
\hline 18 & 0.560 & 0.559 & 0.557 & 0.574 & 0.573 & 0.571 & 0.588 & 0.586 & 0.585 \\
\hline 19 & 0.537 & 0.536 & 0.535 & 0.545 & 0.544 & 0.543 & 0.553 & 0.552 & 0.551 \\
\hline 20 & 0.515 & 0.514 & 0.514 & 0.518 & 0.517 & 0.517 & 0.521 & 0.521 & 0.520 \\
\hline
\end{tabular}

${ }^{\mathrm{a}}$ Contribution ratio $=$ contribution/normal cost; initial fund ratio $=$ initial fund asset/accrual liability.

- Salary scale and inflation rate: $3.5 \%$ for annual salary increase and 3\% for annual inflation rate.

- Discount rate: $6 \%$.

In our model, we assume that $\alpha_{1, t}=(1.06)^{-t}, \alpha_{2, t+1}=(1.06)^{-t} \beta_{2, t+1}, \alpha_{3, t}=(1.06)^{-t} \beta_{3, t}$, and $\alpha_{4, t+1}=$ $(1.06)^{-t} \beta_{4, t+1} \cdot{ }^{6} \beta$ 's are the relative importance among risks to a fund manager. All of them are positive number or zero.

In order to simulate the optimal contribution, we need other assumptions as follows:

- Target fund ratio: $\eta=100 \%$ for every year.

- Risk measurement weight: $\beta_{2, t}=1$ for every year. $\beta_{3, \mathrm{t}}$ and $\beta_{4, \mathrm{t}}$ are parameters in our simulation, and they are set to be $0,0.5$ and 1 . If there is no corresponding risk term $\beta_{3, t}$ and $\beta_{4, t}$ are both zero. $\beta_{3, t}=0.5,1$ and $\beta_{4, t}=0$ are for the case of over-contributing risk. $\beta_{3, t}=0$ and $\beta_{4, t}=0.5,1$ are for the case of under-funding risk.

- Annual investment return process: $r_{t+1}=\theta+\kappa\left(r_{t}-\theta\right)+\varepsilon_{t}$, where $\varepsilon_{t} \sim N\left(0, \sigma^{2} r_{t}\right)$. We assume $\theta=5.946 \%$, $\sigma=2.855 \%$ and $\kappa=0.5744^{7}$ in weekly data. We assume that the fund manager set up their investment plan each year.

- Initial fund ratio: $0.8,1.0$ and 1.2.

- Simulations: $1000{ }^{8}$

We can find that managers who are more concerned with over-contributing risk will make smaller contributions in every period (Table 1). For example, in the case of $\beta_{4}=0.5$ in Table 1 , we find that contribution ratios decrease as $\beta_{3}$ increases no matter what the initial fund ratio is. On the other hand, those who care more about under-funding risk will make larger contributions on every period (Table 1). First part of Table 1 is an example to illustrate this

\footnotetext{
${ }^{6}$ For the purpose of comparison, $v^{t}$ is measured by the inverse of one plus discount rate to the power of time as did in Chang (1999).

7 These parameters are referred from Yeh and Lin (1998). They estimate the term structure of interest rates in Taiwan's bond market by the state space model.

8 The main findings also hold under 500 times of simulation.
} 
Table 2

Decomposition of contributions when initial fund ratio $=1.0^{\mathrm{a}}$

\begin{tabular}{|c|c|c|c|c|c|c|c|c|c|}
\hline \multirow[t]{2}{*}{ Time } & \multicolumn{3}{|c|}{$\beta_{3}=0 ; \beta_{4}=0$} & \multicolumn{3}{|c|}{$\beta_{3}=0 ; \beta_{4}=0.5$} & \multicolumn{3}{|c|}{$\beta_{3}=0 ; \beta_{4}=1.0$} \\
\hline & $\begin{array}{l}\text { Long-term } \\
\text { effect }\end{array}$ & $\begin{array}{l}\text { Short-term } \\
\text { effect }\end{array}$ & Net effect & $\begin{array}{l}\text { Long-term } \\
\text { effect }\end{array}$ & $\begin{array}{l}\text { Short-term } \\
\text { effect }\end{array}$ & Net effect & $\begin{array}{l}\text { Long-term } \\
\text { effect }\end{array}$ & $\begin{array}{l}\text { Short-term } \\
\text { effect }\end{array}$ & Net effect \\
\hline 1 & $1.27 \mathrm{E}+08$ & 0 & $1.27 \mathrm{E}+08$ & $1.77 \mathrm{E}+08$ & 16047528 & $1.93 \mathrm{E}+08$ & $2.27 \mathrm{E}+08$ & 32052505 & $2.59 \mathrm{E}+08$ \\
\hline 2 & $1.35 \mathrm{E}+08$ & 0 & $1.35 \mathrm{E}+08$ & $1.82 \mathrm{E}+08$ & 11881634 & $1.94 \mathrm{E}+08$ & $2.28 \mathrm{E}+08$ & 23772652 & $2.52 \mathrm{E}+08$ \\
\hline 3 & $1.36 \mathrm{E}+08$ & 0 & $1.36 \mathrm{E}+08$ & $1.8 \mathrm{E}+08$ & 9514021 & $1.89 \mathrm{E}+08$ & $2.25 \mathrm{E}+08$ & 19003689 & $2.44 \mathrm{E}+08$ \\
\hline 4 & $1.32 \mathrm{E}+08$ & 0 & $1.32 \mathrm{E}+08$ & $1.73 \mathrm{E}+08$ & 7838673 & $1.81 \mathrm{E}+08$ & $2.14 \mathrm{E}+08$ & 15691885 & $2.29 \mathrm{E}+08$ \\
\hline 5 & $1.27 \mathrm{E}+08$ & 0 & $1.27 \mathrm{E}+08$ & $1.64 \mathrm{E}+08$ & 6566336 & $1.7 \mathrm{E}+08$ & $2.02 \mathrm{E}+08$ & 13120676 & $2.15 \mathrm{E}+08$ \\
\hline 6 & $1.2 \mathrm{E}+08$ & 0 & $1.2 \mathrm{E}+08$ & $1.53 \mathrm{E}+08$ & 5547097 & $1.59 \mathrm{E}+08$ & $1.87 \mathrm{E}+08$ & 11088440 & $1.98 \mathrm{E}+08$ \\
\hline 7 & $1.13 \mathrm{E}+08$ & 0 & $1.13 \mathrm{E}+08$ & $1.43 \mathrm{E}+08$ & 4812458 & $1.48 \mathrm{E}+08$ & $1.74 \mathrm{E}+08$ & 9626688 & $1.84 \mathrm{E}+08$ \\
\hline 8 & $1.04 \mathrm{E}+08$ & 0 & $1.04 \mathrm{E}+08$ & $1.31 \mathrm{E}+08$ & 4207395 & $1.35 \mathrm{E}+08$ & $1.59 \mathrm{E}+08$ & 8409704 & $1.67 \mathrm{E}+08$ \\
\hline 9 & 94265423 & 0 & 94265423 & $1.18 \mathrm{E}+08$ & 3677592 & $1.22 \mathrm{E}+08$ & $1.42 \mathrm{E}+08$ & 7379374 & $1.49 \mathrm{E}+08$ \\
\hline 10 & 83233219 & 0 & 83233219 & $1.04 \mathrm{E}+08$ & 3297004 & $1.07 \mathrm{E}+08$ & $1.25 \mathrm{E}+08$ & 6588479 & $1.32 \mathrm{E}+08$ \\
\hline 11 & 72467832 & 0 & 72467832 & 90280188 & 2927378 & 93207566 & $1.08 \mathrm{E}+08$ & 5865415 & $1.14 \mathrm{E}+08$ \\
\hline 12 & 62011077 & 0 & 62011077 & 76818510 & 2647597 & 79466107 & 91986624 & 5302581 & 97289205 \\
\hline 13 & 51970788 & 0 & 51970788 & 64494524 & 2362281 & 66856805 & 76998565 & 4727924 & 81726489 \\
\hline 14 & 43014672 & 0 & 43014672 & 53247543 & 2145587 & 55393130 & 63488554 & 4289050 & 67777604 \\
\hline 15 & 34434668 & 0 & 34434668 & 42663794 & 1950974 & 44614768 & 50777079 & 3899340 & 54676419 \\
\hline 16 & 26467464 & 0 & 26467464 & 32788173 & 1772818 & 34560991 & 38953668 & 3547350 & 42501018 \\
\hline 17 & 19055459 & 0 & 19055459 & 23609521 & 1642952 & 25252473 & 28104839 & 3284270 & 31389109 \\
\hline 18 & 12060181 & 0 & 12060181 & 15007798 & 1517286 & 16525084 & 17836148 & 3037287 & 20873435 \\
\hline 19 & 5733114 & 0 & 5733114 & 7114743 & 1430109 & 8544852 & 8499979 & 2852979 & 11352957 \\
\hline \multirow[t]{3}{*}{20} & 0 & 0 & 0 & 0 & 1331542 & 1331542 & 0 & 2663345 & 2663345 \\
\hline & \multicolumn{3}{|c|}{$\beta_{3}=0.5 ; \beta_{4}=0$} & \multicolumn{3}{|c|}{$\beta_{3}=0.5 ; \beta_{4}=0.5$} & \multicolumn{3}{|c|}{$\beta_{3}=0.5 ; \beta_{4}=1.0$} \\
\hline & $\begin{array}{l}\text { Long-term } \\
\text { effect }\end{array}$ & $\begin{array}{l}\text { Short-term } \\
\text { effect }\end{array}$ & Net effect & $\begin{array}{l}\text { Long-term } \\
\text { effect }\end{array}$ & $\begin{array}{l}\text { Short-term } \\
\text { effect }\end{array}$ & Net effect & $\begin{array}{l}\text { Long-term } \\
\text { effect }\end{array}$ & $\begin{array}{l}\text { Short-term } \\
\text { effect }\end{array}$ & Net effect \\
\hline 1 & $1.57 \mathrm{E}+08$ & $-5.2 \mathrm{E}+07$ & $1.05 \mathrm{E}+08$ & $2.07 \mathrm{E}+08$ & $-3.6 \mathrm{E}+07$ & $1.71 \mathrm{E}+08$ & $2.56 \mathrm{E}+08$ & $-2 \mathrm{E}+07$ & $2.36 \mathrm{E}+08$ \\
\hline 2 & $1.59 \mathrm{E}+08$ & $-5.4 \mathrm{E}+07$ & $1.05 \mathrm{E}+08$ & $2.06 \mathrm{E}+08$ & $-4.2 \mathrm{E}+07$ & $1.64 \mathrm{E}+08$ & $2.53 \mathrm{E}+08$ & $-3 \mathrm{E}+07$ & $2.23 \mathrm{E}+08$ \\
\hline 3 & $1.56 \mathrm{E}+08$ & $-5.5 \mathrm{E}+07$ & $1 \mathrm{E}+08$ & $2.01 \mathrm{E}+08$ & $-4.6 \mathrm{E}+07$ & $1.55 \mathrm{E}+08$ & $2.45 \mathrm{E}+08$ & $-3.6 \mathrm{E}+07$ & $2.08 \mathrm{E}+08$ \\
\hline 4 & $1.49 \mathrm{E}+08$ & $-5.6 \mathrm{E}+07$ & 92260098 & $1.9 \mathrm{E}+08$ & $-4.8 \mathrm{E}+07$ & $1.41 \mathrm{E}+08$ & $2.31 \mathrm{E}+08$ & $-4.1 \mathrm{E}+07$ & $1.9 \mathrm{E}+08$ \\
\hline 5 & $1.4 \mathrm{E}+08$ & $-5.7 \mathrm{E}+07$ & 83800954 & $1.78 \mathrm{E}+08$ & $-5 \mathrm{E}+07$ & $1.28 \mathrm{E}+08$ & $2.16 \mathrm{E}+08$ & $-4.4 \mathrm{E}+07$ & $1.72 \mathrm{E}+08$ \\
\hline 6 & $1.31 \mathrm{E}+08$ & $-5.7 \mathrm{E}+07$ & 74099511 & $1.65 \mathrm{E}+08$ & $-5.1 \mathrm{E}+07$ & $1.13 \mathrm{E}+08$ & $1.98 \mathrm{E}+08$ & $-4.6 \mathrm{E}+07$ & $1.52 \mathrm{E}+08$ \\
\hline 7 & $1.22 \mathrm{E}+08$ & $-5.7 \mathrm{E}+07$ & 65499179 & $1.53 \mathrm{E}+08$ & $-5.2 \mathrm{E}+07$ & $1 \mathrm{E}+08$ & $1.83 \mathrm{E}+08$ & $-4.7 \mathrm{E}+07$ & $1.36 \mathrm{E}+08$ \\
\hline 8 & $1.11 \mathrm{E}+08$ & $-5.7 \mathrm{E}+07$ & 54701574 & $1.39 \mathrm{E}+08$ & $-5.3 \mathrm{E}+07$ & 85911579 & $1.65 \mathrm{E}+08$ & $-4.8 \mathrm{E}+07$ & $1.17 \mathrm{E}+08$ \\
\hline 9 & 99782009 & $-5.7 \mathrm{E}+07$ & 43221273 & $1.24 \mathrm{E}+08$ & $-5.3 \mathrm{E}+07$ & 70618098 & $1.47 \mathrm{E}+08$ & $-4.9 \mathrm{E}+07$ & 98035918 \\
\hline 10 & 87466358 & $-5.6 \mathrm{E}+07$ & 31062731 & $1.08 \mathrm{E}+08$ & $-5.3 \mathrm{E}+07$ & 55053184 & $1.29 \mathrm{E}+08$ & $-5 \mathrm{E}+07$ & 79101921 \\
\hline 11 & 75805378 & $-5.6 \mathrm{E}+07$ & 19904561 & 93542093 & $-5.3 \mathrm{E}+07$ & 40580118 & $1.11 \mathrm{E}+08$ & $-5 E+07$ & 61237128 \\
\hline 12 & 64070552 & $-5.5 \mathrm{E}+07$ & 8621404 & 79348605 & $-5.3 \mathrm{E}+07$ & 26545428 & 94154867 & $-5 E+07$ & 44012781 \\
\hline 13 & 53746806 & $-5.5 \mathrm{E}+07$ & -1071918 & 66098557 & $-5.2 \mathrm{E}+07$ & 13643922 & 78704640 & $-5 E+07$ & 28608925 \\
\hline 14 & 44088045 & $-5.4 \mathrm{E}+07$ & $-1 \mathrm{E}+07$ & 54338125 & $-5.2 \mathrm{E}+07$ & 2087157 & 64515680 & $-5 \mathrm{E}+07$ & 14409472 \\
\hline 15 & 35269403 & $-5.4 \mathrm{E}+07$ & $-1.9 \mathrm{E}+07$ & 43328070 & $-5.2 \mathrm{E}+07$ & -8574038 & 51371060 & $-5 E+07$ & 1423434 \\
\hline 16 & 26939829 & $-5.3 \mathrm{E}+07$ & $-2.6 \mathrm{E}+07$ & 33107939 & $-5.1 \mathrm{E}+07$ & $-1.8 \mathrm{E}+07$ & 39463270 & $-5 \mathrm{E}+07$ & $-1 \mathrm{E}+07$ \\
\hline 17 & 19379115 & $-5.3 \mathrm{E}+07$ & $-3.3 \mathrm{E}+07$ & 23791296 & $-5.1 \mathrm{E}+07$ & $-2.7 \mathrm{E}+07$ & 28239573 & $-5 \mathrm{E}+07$ & $-2.1 E+07$ \\
\hline 18 & 12218569 & $-5.2 \mathrm{E}+07$ & $-4 \mathrm{E}+07$ & 15076243 & $-5.1 \mathrm{E}+07$ & $-3.6 \mathrm{E}+07$ & 17969760 & $-4.9 \mathrm{E}+07$ & $-3.1 E+07$ \\
\hline 19 & 5771557 & $-5.1 \mathrm{E}+07$ & $-4.6 \mathrm{E}+07$ & 7130044 & $-5 \mathrm{E}+07$ & $-4.3 \mathrm{E}+07$ & 8519714 & $-4.9 \mathrm{E}+07$ & $-4 \mathrm{E}+07$ \\
\hline 20 & 0 & $-5.1 \mathrm{E}+07$ & $-5.1 \mathrm{E}+07$ & 0 & $-4.9 \mathrm{E}+07$ & $-4.9 \mathrm{E}+07$ & 0 & $-4.8 \mathrm{E}+07$ & $-4.8 \mathrm{E}+07$ \\
\hline
\end{tabular}


Table 2 (Continued)

\begin{tabular}{|c|c|c|c|c|c|c|c|c|c|}
\hline \multirow[t]{2}{*}{ Time } & \multicolumn{3}{|c|}{$\beta_{3}=1.0 ; \beta_{4}=0$} & \multicolumn{3}{|c|}{$\beta_{3}=1.0 ; \beta_{4}=0.5$} & \multicolumn{3}{|c|}{$\beta_{3}=1.0 ; \beta_{4}=1.0$} \\
\hline & $\begin{array}{l}\text { Long-term } \\
\text { effect }\end{array}$ & $\begin{array}{l}\text { Short-term } \\
\text { effect }\end{array}$ & Net effect & $\begin{array}{l}\text { Long-term } \\
\text { effect }\end{array}$ & $\begin{array}{l}\text { Short-term } \\
\text { effect }\end{array}$ & Net effect & $\begin{array}{l}\text { Long-term } \\
\text { effect }\end{array}$ & $\begin{array}{l}\text { Short-term } \\
\text { effect }\end{array}$ & Net effect \\
\hline 1 & $1.85 \mathrm{E}+08$ & $-1.03 \mathrm{E}+08$ & 82054949 & $2.36 \mathrm{E}+08$ & $-8.7 \mathrm{E}+07$ & $1.48 \mathrm{E}+08$ & $2.85 \mathrm{E}+08$ & $-7.1 \mathrm{E}+07$ & $2.14 \mathrm{E}+08$ \\
\hline 2 & $1.83 \mathrm{E}+08$ & $-1.08 \mathrm{E}+08$ & 75516122 & $2.3 \mathrm{E}+08$ & $-9.6 \mathrm{E}+07$ & $1.34 \mathrm{E}+08$ & $2.77 \mathrm{E}+08$ & $-8.4 \mathrm{E}+07$ & $1.93 \mathrm{E}+08$ \\
\hline 3 & $1.77 \mathrm{E}+08$ & $-1.11 \mathrm{E}+08$ & 66050962 & $2.21 \mathrm{E}+08$ & $-1.01 \mathrm{E}+08$ & $1.2 \mathrm{E}+08$ & $2.66 \mathrm{E}+08$ & $-9.2 \mathrm{E}+07$ & $1.74 \mathrm{E}+08$ \\
\hline 4 & $1.66 \mathrm{E}+08$ & $-1.13 \mathrm{E}+08$ & 53567124 & $2.07 \mathrm{E}+08$ & $-1.05 \mathrm{E}+08$ & $1.02 \mathrm{E}+08$ & $2.48 \mathrm{E}+08$ & $-9.7 \mathrm{E}+07$ & $1.51 \mathrm{E}+08$ \\
\hline 5 & $1.55 \mathrm{E}+08$ & $-1.13 \mathrm{E}+08$ & 41869975 & $1.92 \mathrm{E}+08$ & $-1.07 \mathrm{E}+08$ & 85656600 & $2.29 \mathrm{E}+08$ & $-1.00 \mathrm{E}+08$ & $1.29 \mathrm{E}+08$ \\
\hline 6 & $1.42 \mathrm{E}+08$ & $-1.13 \mathrm{E}+08$ & 29032870 & $1.76 \mathrm{E}+08$ & $-1.08 \mathrm{E}+08$ & 68097143 & $2.09 \mathrm{E}+08$ & $-1.02 \mathrm{E}+08$ & $1.07 \mathrm{E}+08$ \\
\hline 7 & $1.32 \mathrm{E}+08$ & $-1.14 \mathrm{E}+08$ & 17871859 & $1.62 \mathrm{E}+08$ & $-1.09 \mathrm{E}+08$ & 52990292 & $1.92 \mathrm{E}+08$ & $-1.04 \mathrm{E}+08$ & 87835072 \\
\hline 8 & $1.19 \mathrm{E}+08$ & $-1.14 \mathrm{E}+08$ & 5367216 & $1.46 \mathrm{E}+08$ & $-1.09 \mathrm{E}+08$ & 36411768 & $1.73 \mathrm{E}+08$ & $-1.05 \mathrm{E}+08$ & 67456907 \\
\hline 9 & $1.05 \mathrm{E}+08$ & $-1.13 \mathrm{E}+08$ & -7832186 & $1.29 \mathrm{E}+08$ & $-1.09 \mathrm{E}+08$ & 19796037 & $1.53 \mathrm{E}+08$ & $-1.06 \mathrm{E}+08$ & 46985448 \\
\hline 10 & 91853447 & $-1.13 \mathrm{E}+08$ & $-2.1 \mathrm{E}+07$ & $1.13 \mathrm{E}+08$ & $-1.10 \mathrm{E}+08$ & 3172811 & $1.33 \mathrm{E}+08$ & $-1.06 \mathrm{E}+08$ & 27101028 \\
\hline 11 & 78794794 & $-1.12 \mathrm{E}+08$ & $-3.3 \mathrm{E}+07$ & 96667696 & $-1.09 \mathrm{E}+08$ & $-1.2 \mathrm{E}+07$ & $1.15 \mathrm{E}+08$ & $-1.06 \mathrm{E}+08$ & 8898112 \\
\hline 12 & 66502303 & $-1.11 \mathrm{E}+08$ & $-4.4 \mathrm{E}+07$ & 81589144 & $-1.08 \mathrm{E}+08$ & $-2.7 \mathrm{E}+07$ & 96540764 & $-1.06 \mathrm{E}+08$ & -9051807 \\
\hline 13 & 55206247 & $-1.10 \mathrm{E}+08$ & $-5.4 \mathrm{E}+07$ & 67893911 & $-1.07 \mathrm{E}+08$ & $-3.9 \mathrm{E}+07$ & 80545850 & $-1.05 \mathrm{E}+08$ & $-2.4 \mathrm{E}+07$ \\
\hline 14 & 45142915 & $-1.09 \mathrm{E}+08$ & $-6.4 \mathrm{E}+07$ & 55471831 & $-1.07 \mathrm{E}+08$ & $-5.1 \mathrm{E}+07$ & 65617110 & $-1.04 \mathrm{E}+08$ & $-3.9 \mathrm{E}+07$ \\
\hline 15 & 35941396 & $-1.08 \mathrm{E}+08$ & $-7.2 \mathrm{E}+07$ & 44063782 & $-1.06 \mathrm{E}+08$ & $-6.2 \mathrm{E}+07$ & 52446101 & $-1.04 \mathrm{E}+08$ & $-5.1 \mathrm{E}+07$ \\
\hline 16 & 27320209 & $-1.06 \mathrm{E}+08$ & $-7.9 \mathrm{E}+07$ & 33721663 & $-1.05 \mathrm{E}+08$ & $-7.1 \mathrm{E}+07$ & 39835872 & $-1.03 E+08$ & $-6.3 \mathrm{E}+07$ \\
\hline 17 & 19575261 & $-1.06 \mathrm{E}+08$ & $-8.6 \mathrm{E}+07$ & 24095009 & $-1.04 \mathrm{E}+08$ & $-8 \mathrm{E}+07$ & 28621501 & $-1.02 \mathrm{E}+08$ & $-7.4 \mathrm{E}+07$ \\
\hline 18 & 12316560 & $-1.04 \mathrm{E}+08$ & $-9.2 \mathrm{E}+07$ & 15227873 & $-1.03 \mathrm{E}+08$ & $-8.8 \mathrm{E}+07$ & 18086790 & $-1.01 \mathrm{E}+08$ & $-8.3 \mathrm{E}+07$ \\
\hline 19 & 5798437 & $-1.03 \mathrm{E}+08$ & $-9.7 \mathrm{E}+07$ & 7193906 & $-1.02 \mathrm{E}+08$ & $-9.4 \mathrm{E}+07$ & 8565288 & $-1.00 \mathrm{E}+08$ & $-9.2 \mathrm{E}+07$ \\
\hline 20 & 0 & $-1.01 \mathrm{E}+08$ & $-1.01 \mathrm{E}+08$ & 0 & $-1 \mathrm{E}+08$ & $-1 \mathrm{E}+08$ & 0 & $-9.9 \mathrm{E}+07$ & $-9.9 \mathrm{E}+07$ \\
\hline
\end{tabular}

${ }^{\mathrm{a}}$ Initial fund ratio $=$ initial fund asset/accrued liability; net effect $=$ long-term effect + short-term effect.

finding. In first part of Table 1 , we find that contribution ratios increase as $\beta_{4}$ increases in given initial fund ratio and given period.

In Table 2, we show the decomposition of contributions under the case of initial fund ratio equal to unit. ${ }^{9}$ The long-term effects are the smallest ones in $\beta_{3}=\beta_{4}=0$ compared with other $\beta_{3}$ and $\beta_{4}$ from Table 2 . This shows that whichever kinds of additional risk fund managers care more about in the future. The long-term effect in contribution will be raised to lower future risks. When we want to get rid of future over-contributing/under-funding risk, we should make a higher contributing level now. This is the trade-off between now and future. On the other hand, the short-term effect in $\beta_{3}$ and $\beta_{4}$ are totally different. Through Table 2 , we find that short-term effects are negative and decrease as $\beta_{3}$ increases. Lower current contribution can prevent current over-contributing risk. This results in a negative short-term effect for $\beta_{3}$. In first part of Table 2 , we can find short-term effects increase as $\beta_{4}$ increases. First and second part of Table 2 indicate the same thing. Higher current contribution can reach a higher fund level and avoid current under-funding risk, resulting in a positive short-term effect for $\beta_{4}$.

The fund ratios in $\beta_{3} \neq 0$ are smaller than those in $\beta_{3}=0$ case (Table 3). A positive $\beta_{3}$ will cause lower contributions. When we contribute less, we get lower fund ratios. In Table 3, we find that fund ratios increase as $\beta_{4}$ increases no matter what the initial fund ratio is. This is because a positive $\beta_{4}$ leads to a higher contribution.

In Table 3, we find that the fund ratios are about the same level in the final period given other conditions equal no matter what the initial fund ratio is. Thus, determining the parameters is very important in pension fund management. For example, when the investment returns are very low, under-funding risk should be greatly concerned. In our simulation, the average annual return is 0.05946 and the discount rate is 0.06 . This is just the case when fund managers should care more about under-funding risk. If fund managers do choose a positive $\beta_{4}$, pension funds can reach a higher fund ratio and the probability of insolvency can be reduced.

\footnotetext{
${ }^{9}$ Long-term and short-term effect of $\beta_{3}$ or $\beta_{4}$ do not depend on $F_{t}$. Thus, we just show one case for illustration.
} 
Table 3

Fund ratios and $\beta$ (for initial fund ratios of $0.8,1.0,1.2)^{\mathrm{a}}$

\begin{tabular}{|c|c|c|c|c|c|c|c|c|c|}
\hline \multirow[t]{2}{*}{ Time } & \multicolumn{3}{|c|}{$\beta_{3}=0 ; \beta_{4}=0$} & \multicolumn{3}{|c|}{$\beta_{3}=0 ; \beta_{4}=0.5$} & \multicolumn{3}{|c|}{$\beta_{3}=0 ; \beta_{4}=1.0$} \\
\hline & 0.8 & 1.0 & 1.2 & 0.8 & 1.0 & 1.2 & 0.8 & 1.0 & 1.2 \\
\hline 1 & 0.800 & 1.000 & 1.200 & 0.800 & 1.000 & 1.200 & 0.800 & 1.000 & 1.200 \\
\hline 2 & 0.888 & 1.010 & 1.137 & 0.969 & 1.092 & 1.217 & 1.052 & 1.174 & 1.299 \\
\hline 3 & 0.954 & 1.041 & 1.131 & 1.067 & 1.155 & 1.240 & 1.181 & 1.264 & 1.355 \\
\hline 4 & 0.968 & 1.038 & 1.104 & 1.096 & 1.165 & 1.231 & 1.222 & 1.290 & 1.355 \\
\hline 5 & 0.979 & 1.036 & 1.089 & 1.113 & 1.170 & 1.222 & 1.245 & 1.301 & 1.356 \\
\hline 6 & 0.999 & 1.048 & 1.094 & 1.138 & 1.185 & 1.230 & 1.271 & 1.322 & 1.368 \\
\hline 7 & 1.006 & 1.048 & 1.087 & 1.140 & 1.185 & 1.224 & 1.276 & 1.320 & 1.361 \\
\hline 8 & 1.016 & 1.055 & 1.090 & 1.153 & 1.191 & 1.228 & 1.287 & 1.325 & 1.363 \\
\hline 9 & 1.013 & 1.050 & 1.083 & 1.150 & 1.185 & 1.221 & 1.284 & 1.319 & 1.352 \\
\hline 10 & 1.000 & 1.034 & 1.063 & 1.134 & 1.167 & 1.199 & 1.266 & 1.299 & 1.332 \\
\hline 11 & 0.982 & 1.013 & 1.041 & 1.114 & 1.144 & 1.176 & 1.246 & 1.280 & 1.309 \\
\hline 12 & 0.973 & 1.001 & 1.029 & 1.103 & 1.133 & 1.163 & 1.234 & 1.267 & 1.296 \\
\hline 13 & 0.961 & 0.990 & 1.014 & 1.092 & 1.118 & 1.149 & 1.221 & 1.254 & 1.283 \\
\hline 14 & 0.945 & 0.973 & 0.996 & 1.074 & 1.099 & 1.131 & 1.203 & 1.235 & 1.262 \\
\hline 15 & 0.931 & 0.959 & 0.979 & 1.058 & 1.083 & 1.112 & 1.186 & 1.218 & 1.244 \\
\hline 16 & 0.918 & 0.946 & 0.968 & 1.046 & 1.070 & 1.098 & 1.172 & 1.203 & 1.233 \\
\hline 17 & 0.903 & 0.930 & 0.950 & 1.029 & 1.052 & 1.082 & 1.155 & 1.184 & 1.215 \\
\hline 18 & 0.883 & 0.911 & 0.929 & 1.009 & 1.033 & 1.062 & 1.135 & 1.166 & 1.197 \\
\hline 19 & 0.863 & 0.892 & 0.910 & 0.990 & 1.016 & 1.046 & 1.116 & 1.150 & 1.182 \\
\hline 20 & 0.854 & 0.883 & 0.902 & 0.983 & 1.011 & 1.043 & 1.111 & 1.150 & 1.181 \\
\hline \multirow[t]{3}{*}{21} & 0.831 & 0.859 & 0.879 & 0.963 & 0.993 & 1.024 & 1.093 & 1.132 & 1.164 \\
\hline & \multicolumn{3}{|c|}{$\beta_{3}=0.5 ; \beta_{4}=0$} & \multicolumn{3}{|c|}{$\beta_{3}=0.5 ; \beta_{4}=0.5$} & \multicolumn{3}{|c|}{$\beta_{3}=0.5 ; \beta_{4}=1.0$} \\
\hline & 0.8 & 1.0 & 1.2 & 0.8 & 1.0 & 1.2 & 0.8 & 1.0 & 1.2 \\
\hline 1 & 0.800 & 1.000 & 1.200 & 0.800 & 1.000 & 1.200 & 0.800 & 1.000 & 1.200 \\
\hline 2 & 0.860 & 0.984 & 1.107 & 0.943 & 1.067 & 1.190 & 1.025 & 1.148 & 1.272 \\
\hline 3 & 0.909 & 0.993 & 1.083 & 1.019 & 1.108 & 1.196 & 1.134 & 1.221 & 1.305 \\
\hline 4 & 0.910 & 0.974 & 1.043 & 1.034 & 1.103 & 1.172 & 1.161 & 1.227 & 1.292 \\
\hline 5 & 0.908 & 0.960 & 1.017 & 1.037 & 1.095 & 1.150 & 1.171 & 1.226 & 1.279 \\
\hline 6 & 0.916 & 0.960 & 1.010 & 1.050 & 1.101 & 1.146 & 1.189 & 1.234 & 1.281 \\
\hline 7 & 0.912 & 0.949 & 0.994 & 1.046 & 1.091 & 1.130 & 1.184 & 1.222 & 1.266 \\
\hline 8 & 0.914 & 0.948 & 0.988 & 1.048 & 1.090 & 1.124 & 1.185 & 1.221 & 1.260 \\
\hline 9 & 0.903 & 0.934 & 0.972 & 1.037 & 1.075 & 1.106 & 1.172 & 1.205 & 1.241 \\
\hline 10 & 0.882 & 0.911 & 0.947 & 1.014 & 1.048 & 1.078 & 1.149 & 1.179 & 1.212 \\
\hline 11 & 0.856 & 0.884 & 0.917 & 0.986 & 1.018 & 1.048 & 1.120 & 1.149 & 1.183 \\
\hline 12 & 0.840 & 0.867 & 0.899 & 0.969 & 1.000 & 1.029 & 1.101 & 1.128 & 1.161 \\
\hline 13 & 0.822 & 0.846 & 0.878 & 0.950 & 0.981 & 1.010 & 1.081 & 1.107 & 1.140 \\
\hline 14 & 0.801 & 0.824 & 0.856 & 0.927 & 0.956 & 0.984 & 1.058 & 1.083 & 1.112 \\
\hline 15 & 0.778 & 0.802 & 0.834 & 0.905 & 0.934 & 0.960 & 1.034 & 1.059 & 1.086 \\
\hline 16 & 0.758 & 0.783 & 0.815 & 0.886 & 0.914 & 0.940 & 1.013 & 1.038 & 1.067 \\
\hline 17 & 0.736 & 0.759 & 0.792 & 0.863 & 0.890 & 0.916 & 0.989 & 1.015 & 1.042 \\
\hline 18 & 0.708 & 0.733 & 0.767 & 0.835 & 0.865 & 0.889 & 0.960 & 0.986 & 1.017 \\
\hline 19 & 0.682 & 0.705 & 0.739 & 0.810 & 0.838 & 0.861 & 0.936 & 0.962 & 0.991 \\
\hline 20 & 0.660 & 0.685 & 0.719 & 0.792 & 0.819 & 0.844 & 0.922 & 0.948 & 0.976 \\
\hline 21 & 0.626 & 0.652 & 0.686 & 0.759 & 0.788 & 0.813 & 0.894 & 0.920 & 0.949 \\
\hline
\end{tabular}


Table 3 (Continued)

\begin{tabular}{|c|c|c|c|c|c|c|c|c|c|}
\hline \multirow[t]{2}{*}{ Time } & \multicolumn{3}{|c|}{$\beta_{3}=1.0 ; \beta_{4}=0$} & \multicolumn{3}{|c|}{$\beta_{3}=1.0 ; \beta_{4}=0.5$} & \multicolumn{3}{|c|}{$\beta_{3}=1.0 ; \beta_{4}=1.0$} \\
\hline & 0.8 & 1.0 & 1.2 & 0.8 & 1.0 & 1.2 & 0.8 & 1.0 & 1.2 \\
\hline 1 & 0.800 & 1.000 & 1.200 & 0.800 & 1.000 & 1.200 & 0.800 & 1.000 & 1.200 \\
\hline 2 & 0.830 & 0.956 & 1.081 & 0.916 & 1.039 & 1.161 & 0.996 & 1.119 & 1.243 \\
\hline 3 & 0.859 & 0.947 & 1.037 & 0.975 & 1.058 & 1.146 & 1.083 & 1.172 & 1.261 \\
\hline 4 & 0.844 & 0.914 & 0.983 & 0.974 & 1.039 & 1.107 & 1.097 & 1.167 & 1.232 \\
\hline 5 & 0.830 & 0.887 & 0.942 & 0.967 & 1.020 & 1.076 & 1.098 & 1.154 & 1.209 \\
\hline 6 & 0.827 & 0.877 & 0.926 & 0.967 & 1.014 & 1.061 & 1.102 & 1.150 & 1.201 \\
\hline 7 & 0.817 & 0.858 & 0.901 & 0.955 & 0.994 & 1.037 & 1.089 & 1.131 & 1.177 \\
\hline 8 & 0.812 & 0.849 & 0.886 & 0.950 & 0.984 & 1.023 & 1.083 & 1.120 & 1.164 \\
\hline 9 & 0.795 & 0.830 & 0.861 & 0.931 & 0.962 & 0.997 & 1.062 & 1.095 & 1.135 \\
\hline 10 & 0.766 & 0.800 & 0.828 & 0.901 & 0.930 & 0.962 & 1.031 & 1.059 & 1.098 \\
\hline 11 & 0.731 & 0.764 & 0.791 & 0.867 & 0.894 & 0.925 & 0.999 & 1.023 & 1.058 \\
\hline 12 & 0.709 & 0.739 & 0.768 & 0.843 & 0.867 & 0.898 & 0.976 & 0.998 & 1.032 \\
\hline 13 & 0.682 & 0.711 & 0.739 & 0.816 & 0.840 & 0.871 & 0.948 & 0.967 & 1.003 \\
\hline 14 & 0.655 & 0.683 & 0.711 & 0.786 & 0.812 & 0.840 & 0.917 & 0.937 & 0.971 \\
\hline 15 & 0.628 & 0.655 & 0.680 & 0.757 & 0.781 & 0.810 & 0.887 & 0.906 & 0.937 \\
\hline 16 & 0.603 & 0.629 & 0.654 & 0.732 & 0.754 & 0.784 & 0.861 & 0.881 & 0.910 \\
\hline 17 & 0.575 & 0.599 & 0.625 & 0.704 & 0.725 & 0.754 & 0.832 & 0.849 & 0.879 \\
\hline 18 & 0.541 & 0.564 & 0.590 & 0.669 & 0.690 & 0.717 & 0.796 & 0.815 & 0.844 \\
\hline 19 & 0.504 & 0.528 & 0.554 & 0.635 & 0.655 & 0.682 & 0.764 & 0.782 & 0.810 \\
\hline 20 & 0.472 & 0.496 & 0.524 & 0.607 & 0.627 & 0.654 & 0.738 & 0.757 & 0.785 \\
\hline 21 & 0.426 & 0.451 & 0.479 & 0.565 & 0.585 & 0.611 & 0.699 & 0.718 & 0.746 \\
\hline
\end{tabular}

${ }^{\mathrm{a}}$ Initial fund ratio $=$ initial fund asset/accrual liability; fund ratio $=$ fund asset/accrual liability.

\section{Conclusions}

The paper considers that pension fund managers care more about under-funding than over-funding, and overcontribution than under-contribution. We find that managers who care more about over-contribution risk will reduce the level of contribution and fund ratios. On the other hand, those who care more about under-funding risk will increase the level of contribution and fund ratios.

We decompose the contribution into three parts to distinguish how these risks affect the contribution. We find that both over-contribution and under-funding risk will raise the long-term effect. On the other hand, the short-term effect is negative when the model involves over-contribution risk, but the short-term effect is positive when the model involves under-funding risk.

\section{Acknowledgements}

The authors are grateful for the valuable comments received from an anonymous referee and the editor.

\section{References}

Bacinello, A.R., 1988. A stochastic simulation procedure for pension scheme. Insurance: Mathematics and Economics 7, $153-161$.

Benjamin, S., 1984. An actuarial layman looks at control theory. In: Proceedings of the Transactions of 22nd International Congress of Actuaries, pp. 295-310.

Benjamin, S., 1989. Driving the pension fund. Journal of Institute of Actuaries 116, 717-735.

Bowers, N.L., Gerber, H.U., Hickman, J.C., Jones, D.A., Nesbitt, C.J., 1982. Notes on the dynamics of pension funding. Insurance: Mathematics and Economics 1, 261-270.

Cairns, A.J., Parker, G., 1997. Stochastic pension fund modeling. Insurance: Mathematics and Economics 21, 43-79. 
Chang, S.C., 1999. Optimal pension funding through dynamic simulations: the case of Taiwan public employees retirement system. Insurance: Mathematics and Economics 24, 187-199.

Chang, S.C., 2000. Realistic pension funding: a stochastic approach. Journal of Actuarial Practice 8, 5-42.

Chang, S.C., Chen, C.C., 2002. Allocating unfunded liability in pension valuation under uncertainty. Insurance: Mathematics and Economics 30, 371-387.

Chang, S.C., Cheng, H.Y., 2002. Pension valuation under uncertainties: implementation of a stochastic and dynamic monitoring system. Journal of Risk and Insurance 69, 171-192.

Cox, J.C., Ingersoll, J.E., Ross, S.A., 1985. An intertemporal general equilibrium model of asset prices. Econometrica 53 (2), $363-384$.

Daykin, C.D., Pentikainen, T., Pesonen, M., 1994. Practical Risk Theory for Actuaries, Monographs on Statistics and Applied Probability, vol. 53. Chapman \& Hall, London, UK.

Dufresne, D., 1988. Moments of pension fund contributions and fund levels when rates of return are random. Journal of the Institute of Actuaries $115,535-544$.

Dufresne, D., 1989. Stability of pension systems when rates of return are random. Insurance: Mathematics and Economics 6, 129-134.

Gerrard, R.J., Haberman, S., 1996. Stability of pension systems when gains/losses are amortized and rates of return are autoregressive. Insurance: Mathematics and Economics 18, 59-71.

Haberman, S., 1992. Pension funding with time delays: a stochastic approach. Insurance: Mathematics and Economics 11, 179-189.

Haberman, S., 1993. Pension funding with time delays and autoregressive rates of investment return. Insurance: Mathematics and Economics $13,45-56$.

Haberman, S., 1994. Autoregressive rates of return and the variability of pension contributions and fund levels for a defined benefit pension scheme. Insurance: Mathematics and Economics 14, 219-240.

Haberman, S., Sung, J.H., 1994. Dynamic approaches to pension funding. Insurance: Mathematics and Economics 15, $151-162$.

Haberman, S., Wong, L.Y., 1997. Moving average rates of return and the variability of pension contributions and fund levels for a defined benefit pension scheme. Insurance: Mathematics and Economics 20, 115-135.

Mandl, P., Mazurova, L., 1996. Harmonic analysis of pension funding methods. Insurance: Mathematics and Economics 17, $203-214$.

McKenna, F.W., 1982. Pension plan cost risk. Journal of Risk and Insurance 49, 193-217.

O’Brian, T., 1986. A stochastic-dynamic approach to pension funding. Insurance: Mathematics and Economics 5, 141-146.

O'Brian, T., 1987. A two-parameter family of pension contribution functions and stochastic optimization. Insurance: Mathematics and Economics $6,129-134$.

Owadally, M.I., Haberman, S., 1999. Pension fund dynamics and gain/losses due to random rates of investment return. North American Actuarial Journal 3, 105-117.

Owadally, M.I., Haberman, S., 2000. Efficient Amortization of Actuarial Gains/Losses and Optimal Funding in Pension Plans. Actuarial Research Paper No. 133. Department of Actuarial Science and Statistics, City University, London.

Vanderbroek, M., 1990. Pension funding and optimal control. Mitteilungen der schweizerische Vereinigung der Versicherungamathematiker 2, 313-325.

Yeh, S.K., Lin, B.H., 1998. Empirical investigation on the Taiwan term structure of interest rates models: an application of the state space model. Review of Securities and Futures Markets 10, 55-88. 